\title{
GCU
}

Glasgow Caledonian

University

University for the Common Good

\section{Producing uniform illumination within a rectangular area by using a nonimaging optic}

Babadi, Sina; Ramirez-Iniguez, Roberto; Boutaleb, Tuleen; Mallick, Tapas

Published in:

Applied Optics

DOI:

10.1364/AO.57.009357

Publication date:

2018

Document Version

Author accepted manuscript

Link to publication in ResearchOnline

Citation for published version (Harvard):

Babadi, S, Ramirez-Iniguez, R, Boutaleb, T \& Mallick, T 2018, 'Producing uniform illumination within a rectangular area by using a nonimaging optic', Applied Optics, vol. 57, no. 31, pp. 9357-9364.

https://doi.org/10.1364/AO.57.009357

\section{General rights}

Copyright and moral rights for the publications made accessible in the public portal are retained by the authors and/or other copyright owners and it is a condition of accessing publications that users recognise and abide by the legal requirements associated with these rights.

Take down policy

If you believe that this document breaches copyright please view our takedown policy at https://edshare.gcu.ac.uk/id/eprint/5179 for details of how to contact us. 


\title{
Producing uniform illumination within a rectangular area by using a novel non-imaging optic
}

\author{
Sina Babadi, ${ }^{1,}{ }^{*}$ Roberto RAMirez-Inguiez, ${ }^{1}$ TUleEn Boutaleb, ${ }^{1}$ TAPAS \\ MALLICK $^{2}$ \\ ${ }^{1}$ School of Engineering and Built Environment, Glasgow Caledonian University, Glasgow, UK \\ ${ }^{2}$ Environment and Sustainability Institute, University of Exeter, UK \\ *Corresponding author: sina.babadi@gcu.ac.uk
}

Received XX Month XXXX; revised XX Month, XXXX; accepted XX Month XXXX; posted XX Month XXXX (Doc. ID XXXXX); published XX Month XXXX

This paper proposes a new design method to create a novel optical element to generate uniform illumination within a rectangular area. Based on this model, an illuminated area is irradiated by two sets of rays, the first one irradiates the target plane after refraction from the top section of the lens and the second one irradiates from the reflection at the side profile of the lens and then refraction at the top part of the lens. The results show that a uniformity of over $90 \%$ can be achieved.

http://dx.doi.org/10.1364/AO.99.099999

\section{INTRODUCTION}

Light emitting diodes (LEDs) are becoming increasingly important in a variety of applications because of their increasing efficiency (lumen per watt) and to the rapid decline in their production costs [1]. Other features of LEDs that compared to other traditional light sources make them desirable are: a longer life, lower energy consumption, their smaller size and a shorter response time [2]. However, there are still some issues related to the use of LEDs for illumination system that have to be addressed. This is the case, for instance, in applications such as street lighting [3]. This is due to fact that street light luminaires have to meet national and international street lighting standards [4]. The Lambertian radiation pattern of LEDs, for example, can pose a challenge when used for street lighting. This is because they produce a circular illumination footprint with higher light density at the centre and lower light density at the edge of the illuminated area [5]. However, in order to satisfy the standards, the illuminated area should have uniform illumination. Moreover, using an LED (without the aid of an additional optic) for street lighting increases light pollution and eye glare because LEDs irradiate with high divergence. To overcome this problem a secondary optic is proposed to redirect the output beam to the desired illuminated area. The secondary optic can also be used to reshape the illumination footprint.

Different techniques have been used to design secondary optics in order to produce uniform illumination. These techniques include simultaneous multiple surface (SMS) [6-8], partial differential equation and non-uniform rational B-spline (NURBS) methods [9]. In general, a uniformly illuminated area can be achieved by using lenses with either a simple structure (e.g. consisting of a single refractive surface) or with more complex structures (consisting of reflective and refractive surfaces).

Zhenrong Z. et al. [10] designed a lens based on total internal reflection (TIR) at the side profile of the lens. The relationship between the rays at the inside and the outside of the lens was defined using Snell laws. Then, it was solved by using the Runge-Kutta formula. The results demonstrated a 78.6\% optical efficiency (transferring light from the LED to the target plane) and $78.4 \%$ uniformity within a circular area.

$\mathrm{XU}$ et al. [11] designed a smooth freeform lens to produce uniform illumination for road lighting by a single refraction on the surface of the lens. This lens was designed based on the ideal point source, therefore by increasing the size of the light source, uniformity was reduced. Due to the output rays' exit from all over the surface of the lens, it is necessary to provide a gap between these lenses to reduce the effect from neighbouring lenses. Want et al. [12] presented a discontinuous freeform lens to provide uniform illumination within a rectangular area. Using a discontinuous surface makes the size of the lens smaller than a smooth surface lens. However, uniformity is provided by a single refraction on the surface of the lens.

This research proposes a novel energy mapping method to produce an optic. Based on this design uniformity was achieved by transferring a specific amount of light flux to a prescribed area. Section 2 explains concepts related to non-imaging optics and the main characteristics of the Dielectric Totally Internally Reflecting Concentrator (DTIRC) as a concentrator. Then, the use of the DTIRC as an illumination system is explained. Section 3. A introduces the new energy mapping method to produce an optic based on TIR at the side profile of the lens in order to 
produce uniformity within a rectangular area. Finally, in Section 3. B and $\mathrm{C}$, the optical performance obtained from simulations and experimental work are presented, and the use of the new lens to produce uniform illumination is discussed.

\section{NON-IMAGING OPTIC}

Non-imaging optics are a type of optics that transfer light between a light source and a target plane without requiring the creation of an image of the light source at the target [13]. Many different techniques based on refraction and reflection are available for the design of nonimaging optics in order to optimise the radiative transfer from a light source to a target plane. The hemispherical concentrator, the compound parabolic concentrator (CPC) and the dielectric totally internally reflecting concentrator (DTIRC) are examples of nonimaging optics $[14,15]$. Due to the compactness and higher efficiency of the DTIRC, it was selected as a candidate for illumination systems.

The DTIRC comprises of three sections: a curved front surface, a TIR side profile, and an exit aperture. The design of the DTIRC is based on Snell's laws of refraction and reflection, and Fermat's principle. Figure 1 shows a schematic view of a 2-D version of a DTIRC [16]. When used as a concentrator, impinging rays at the top front curve of the DTIRC enter the optic and are refracted within the concentrator. Subsequently they are totally internally reflected at the side wall of the lens and directed to the exit aperture of the lens as long as these rays are within the acceptance angle of the concentrator. The rays incident on the front surface beyond the acceptance angle do not satisfy the condition of TIR at the profile of the DTIRC, and therefore they refract out of the lens.

\section{A. DTIRC in illumination system}

In this section, DTIRC is used in an illumination system to control the output rays of a light source by using TIR at the side profile of it. In this case, a light source was attached to the exit aperture of the DTIRC and light exits from the top section of it. A DTIRC is defined with below details, this DTIRC is used in experiment and simulation with the same characteristics. Ray tracing simulation and its footprint in simulation and experiment validate TIR at the side profile of it and collimating the output rays of the light source.

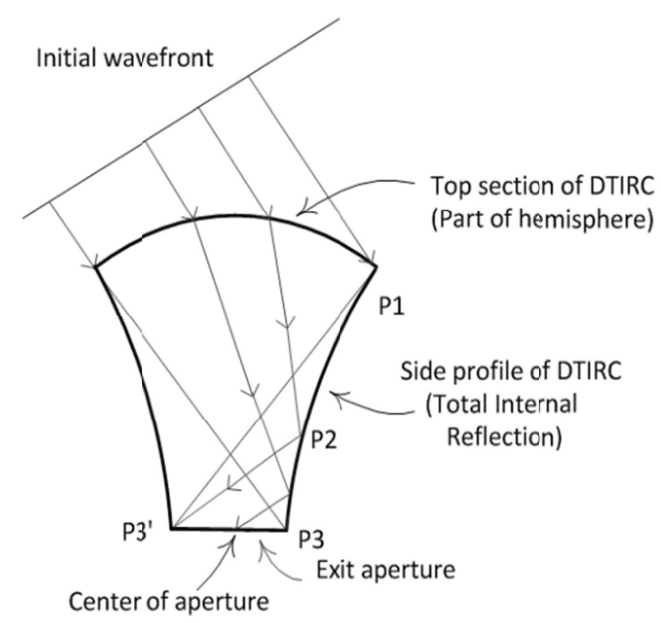

Fig. 1. Schematic view of the ray tracing inside of the DTIRC [16].
The characteristics of the DTIRC used in the experiment and simulation are provided. They are as follows the field-of-view (FOV) is $\pm 1.67^{\circ}$, and the smallest aperture of the DTIRC has a $500 \mu \mathrm{m}$ diameter, matched to the emission area of the LED to redirect all the light emitted from the LED. The total height and the largest aperture diameter of the DTIRC are $6.17 \mathrm{~cm}$ and $2.61 \mathrm{~cm}$, respectively. The photograph of the projection of the LED-DTIRC structure beam onto a white piece of paper, taken at a distance of $0.46 \mathrm{~m}$, is shown in Figure 2. Figure 2 shows (a) the collimating effect after the light from the LED, which originally had a wide angular emission of rays, after passing through the DTIRC; and (b) the light distribution at the target plane obtained experimentally.

Despite controlling the output emission rays by TIR at the side profile of the DTIRC, this optic was unable to produce uniform illumination. Another optic, the circular dielectric totally internally reflecting optic (CDTIRO), was designed to produce uniform illumination by TIR at the side profile. The structure and design process of the CDTIRO is explained in detail in $[17,18]$. The CDTIRO produces uniformity within a circular area by using its side profile while the top section remains as a part of the hemisphere. The angular distribution of the LED is assumed to be Lambertian. The illumination footprint from the CDTIRO has contributions from two sets of rays, the first from rays which irradiate directly through the top section of the lens (for LED rays emitted with angles between 0 and $\theta$ as shown in Figure 3). The second part of the illumination is irradiated from the second set of rays via reflection at the side profile of the lens (for LED rays emitted with angles between $\theta$ and $\phi$ ) and refraction from the top section of the lens, as can be seen in Figure 3 and 4. Figure 3 (a) shows the schematic view of the ray tracing of the first section of the illumination, with the output rays refracted towards the target plane. Figure 3 (b) shows the cross section of the irradiance corresponding to the first set of rays at the target plane, with the highest intensity at the centre of the target plane. The illumination level decreases towards the edge of the illuminated area.

In order to generate uniform illumination, the light intensity of the second set of rays at the edge of the illuminated area should be higher than at the centre of the illuminated area to compensate for the nonuniform illumination of the first set of rays. The illuminated area and the angular range between $\theta$ and $\phi$ were divided into the same number of sections. Each ray after reflection at the side profile of the lens and refraction on the top section of the lens reached the illuminated area. For example, Figure 4 (a) shows five rays between $\theta$ and $\phi$, which after reflection at the side profile reach the corresponding desired points $\left(\mathrm{r}_{0}-\mathrm{r}_{5}\right)$ on the illuminated area. (a)

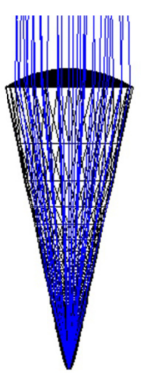

(b)

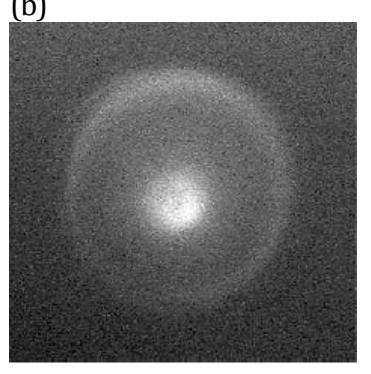

Fig. 2. Collimation and footprint from the LED-DTIRC (a) Ray tracing through the DTIRC combined with an LED obtained from software simulations in ZEMAX. (b) Light distribution on a square target plane of $10(\mathrm{~cm}) \times 10(\mathrm{~cm})$ obtained experimentally [14]. 
(a)

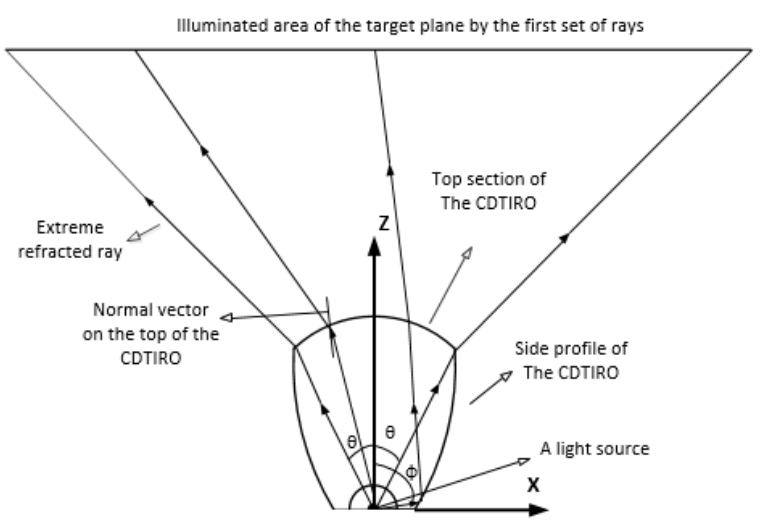

(b)

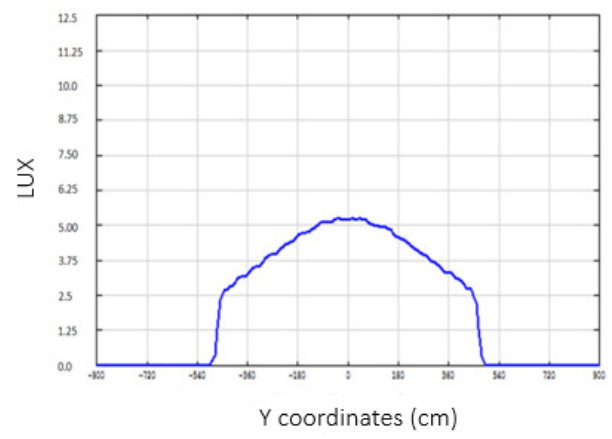

Fig. 3. First set of illumination. a) Schematic view of the ray tracing inside the CDTIRO corresponding to the first set of rays. b) Cross section of light distribution of the first set of rays at the target plane.

The angular distribution of LEDs can be either a Lambertian or nonLambertian. In this research project, a Lambertian model was chosen due to the widespread of the Lambertian radiation pattern of LEDs. However, this method can be used for non-Lambertian radiation pattern of the LEDs. Total amount of energy of any angular distribution of LEDs (Lambertian and non-Lambertian) must be calculated to produce uniform illumination. The position of the desired points on the illuminated area can be calculated using Equation 1:

$\frac{\pi\left(r_{0}^{2}-r_{1}^{2}\right)}{\pi r_{0}^{2}}=\frac{\Omega_{\theta-\theta_{1}} \times \sum_{\theta}^{\theta_{1}} \cos Y}{\Omega_{\theta-\phi} \times \sum_{\theta}^{\phi} \cos X}$

$\Omega$ is a symbol of the solid angle in Eq. 1 . It is used to calculate the amount of energy in the volume. Due to a Lambertian radiation pattern of the light source $\sum_{\theta_{1}}^{\theta_{2}} \cos Y$ and $\sum_{\theta}^{\phi} \cos X$ were added to Eq. 1 . In Eq. $1, \mathrm{Y}$ is the variable angle between $\theta_{1}$ and $\theta_{2}$, and $\mathrm{X}$ is the variable angle between $\theta$ and $\phi$. Figure 4.b shows the cross section of the second set of illumination, which is provided by Eq. 1 to compensate for the nonuniformity of the first set of illumination, thereby producing uniform illumination. The position of the second desired point $\left(\mathrm{r}_{2}\right)$ can be calculated in Eq. 2. Based on this relationship, the position of the last desired point $\left(\mathrm{r}_{5}\right)$ is the center of the illuminated area.

$\frac{\pi\left(r_{1}^{2}-r_{2}^{2}\right)}{\pi r_{0}^{2}}=\frac{\Omega_{\theta_{1}-\theta_{2}} \times \Sigma_{\theta_{1}}^{\theta_{2}} \cos Y}{\Omega_{\theta-\phi} \times \Sigma_{\theta}^{\phi} \cos X}$

$\theta$ can be calculated by following equation:

$\theta=\mu+v=\mu+\arcsin \left(\frac{n_{2} \sin \eta}{n_{1}}\right)$ (a)

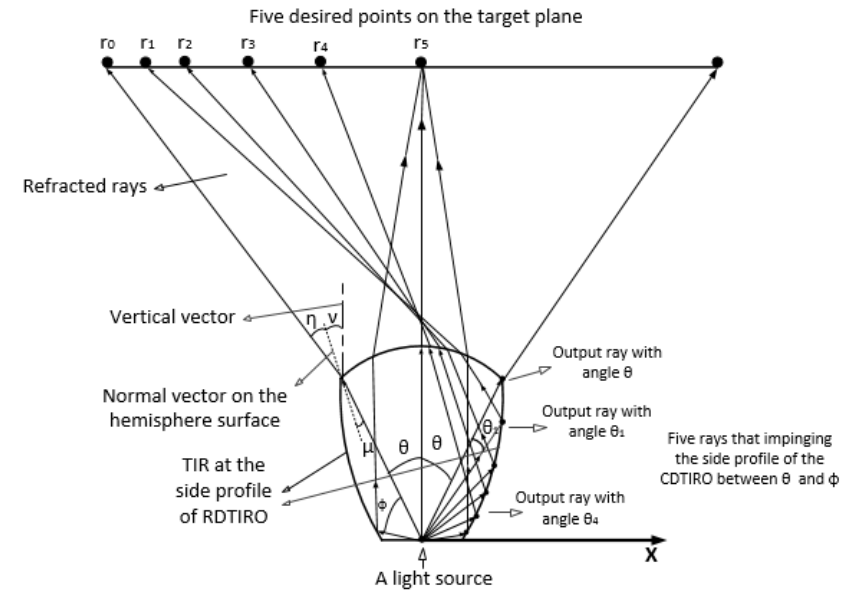

(b)

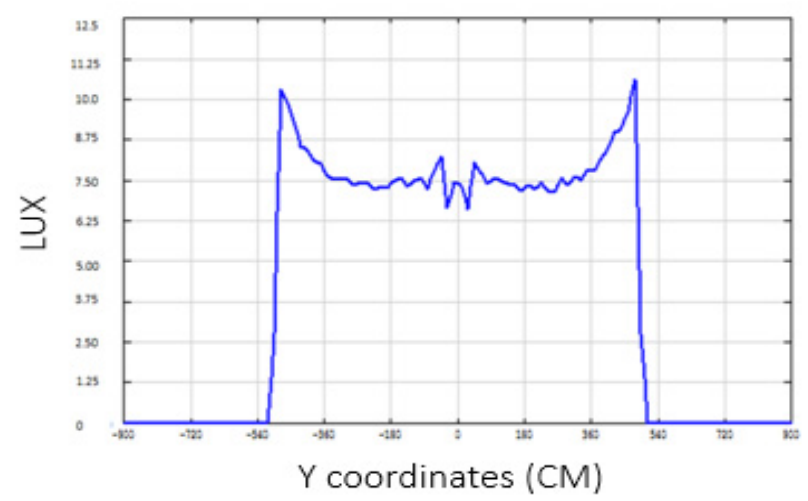

Fig. 4. Second set of illumination. a) Schematic view of the ray tracing inside the CDTIRO corresponding to the $2^{\text {nd }}$ set of rays. b) Cross section of the light distribution at the target plane.

Uniform illumination is generated by a combination of the first and second sets of rays. Figure 5 shows the uniformity created by this method. This model produces over $90 \%$ of uniformity within a circular area.

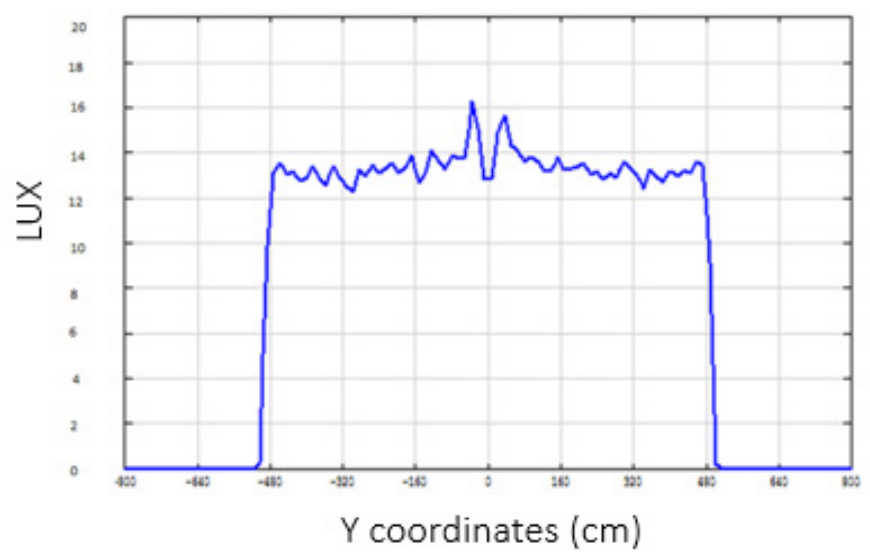

Fig. 5. Uniformity of illuminance at the target plane produced by the combination of both sets of rays. 
This section has described the design process of a CDTIRO to produce uniform illumination within a circular area. However, it is desirable to provide uniform illumination within a rectangular area for street lighting.

\section{DESIGINIG A NOVEL OPTIC TO PRODUCE UNIFORMITY WITHIN A RECTANGULAR AREA}

This section follows on from the previous method (the CDTIRO method) to design the rectangular dielectric total internally reflecting optic (RDTIRO) based on the combination of several CDTIROs sections to produce a rectangular illumination footprint. Therefore, the main concepts and structure of the RDTIRO are similar in those of the CDTIRO. In this section, two more descriptions are added to design a RDTIRO. They include: (i) the introduction of energy mapping for a rectangular area; and (ii) the optimisation on the side profile of the optic to increase uniformity within the illuminated area.

\section{A. Energy mapping to produce uniformity within a rectangular area}

Figure 6 shows a new energy mapping method that is used to design the RDTIRO. As explained above, the concept and the illumination method used to produce this optic are similar to the ones used to create the CDTIRO. This optic illuminates a target plane two times (i.e. there are two sets of rays corresponding to (i) only refraction, and (ii) to reflectionrefraction). As it can be seen in Figure 6, one quarter of this target plane was divided into eight sections $\left(S_{1}\right.$ to $\left.S_{8}\right)$. The $R_{1}$ to $\mathrm{R}_{8}$ and $\mathrm{R}_{1}$ ' to $\mathrm{R}_{8}$ ' are curved lines corresponding to the sections $\mathrm{S}_{1}$ to $\mathrm{S}_{8}$ and sections $\mathrm{S}_{1}$ ' to $\mathrm{S}_{8}$, , respectively.

In this design model, the side profile of the CDTIROs can be designed and calculated based on the length of the $\mathrm{R}$ lines. But these side profiles of the CDTIROs are rotated by an increment of $\delta$ in degrees. For example, the first CDTIRO was rotated by $\delta_{1}$ degrees around the $\mathrm{Z}$-axis on the Y-X plane with the angle measured with respect to the X-Z plane, as shown in Figure 6 . The eight CDTIROs were designed and rotated $\delta$ degrees with respect to their $\mathrm{R}$ lines. The combination of all small section of the CDTIROs generates the RDTIRO, the top section of the RDTIRO remains as a part of the hemisphere and the discontinuous side profile of the RDTIRO is produced by combining discrete CDTIROs sections.

The first set of the rays corresponding to the first quarter of the light source (the rays with angle between 0 and $\theta$ with respect to the Z-axis) refract to sections $\mathrm{S}_{1}$ to $\mathrm{S}_{8}$. This is also the case of the second set of rays of the first quarter at the light source (the rays with angle between $\theta$ and $\phi$ ) which reflect and refract to the sections $\mathrm{S}_{1}{ }^{\prime}$ to $\mathrm{S}_{8}$ ' (the third quarter of the target plane). For example, the first CDTIRO section in the RDTIRO illuminates sections $S_{1}$ and $S_{1}{ }^{\prime}$ in Fig. 6 . In this case, the first set of rays (angle $\theta$ that is between 0 and 27) reaches the $S_{1}$ section, while the second set of rays (the angle between $\theta$ and $\phi$ that is between 27 and 85 ) reaches the $S_{1}{ }^{\prime}$ section. In a similar way, the first set of the third quarter of the light source illuminates the section $S_{1}{ }^{\prime}$ to $S_{8}{ }^{\prime}$ and the second set of rays illuminate the section $S_{1}$ to $S_{8}$.

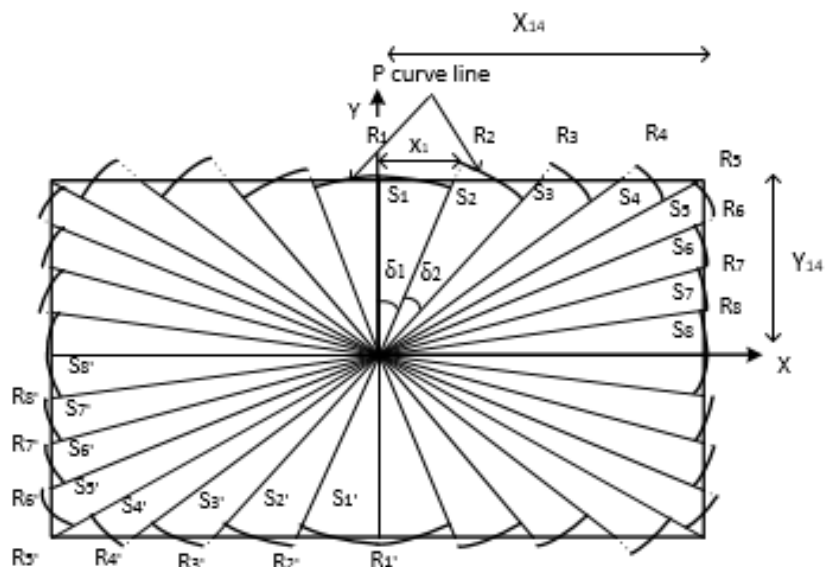

Fig. 6. Energy distribution plan of the target plane, one quarter of the target plane has been divided into 8 equal sections.

In the following example, the RDTIRO was designed for a $7 \mathrm{~m}$ distance from the light source to the target plane to illuminate a rectangular area of $11 \mathrm{~m} \times 7 \mathrm{~m}$. It was assumed that the emission surface of an extended light source was $0.89 \mathrm{~cm}$ diameter with a Lambertian radiation pattern; the maximum emission angle with respect to the Z-axis (the $\phi$ angle) was set to $85^{\circ}$. One quarter of this target plane was divided into 8 equal triangular areas.

In this energy mapping, the $\mathrm{R}$ line of the first section $\left(\mathrm{S}_{1}\right)$ has the smallest length. Therefore, the first angular range $(0$ and $\theta$ angles of section $S_{1}$ ) was calculated to be between 0 and 27 degrees for the top section of the lens. The second angular range $\left(\theta\right.$ and $\phi$ of section $\left.S_{1}\right)$ was then between 27 and 85 degrees for reflection-refraction. The $\mathrm{R}$ line of fifth section has the longest length which corresponded to angle $\theta$ of 39 degrees. The design process of the various CDTIROs is similar to the described above. These CDTIROs sections produce uniform illumination within their triangular areas.

Despite providing uniformity within the individual illuminated sections, this optic cannot provide uniformity on the target plane as shown in Figure 7. This non-uniformity was generated due to each section of the lens (the CDTIRO crosssections of the lens) receiving different amounts of the luminous flux from the light source. As it can be seen in Figure 6 , the angles $\delta$ are different for each triangular area. The angles $\delta$ in the energy mapping have larger variations when the rectangular area becomes narrower.

Table 1 shows the portion of the light flux of each segment of the RDTIRO. This light flux enters the RDTIRO and after refractionreflection reaches the specified section. The section $\mathrm{S}_{1}$ receives $20.44 \%$ of the light flux from the light source, but the section $\mathrm{S}_{5}$ only receives $8.38 \%$ of the light flux. Figure 7 (a) shows the ray tracing simulation of the RDTIRO combined with an extended light source. There is TIR at the side profile of the RDTIRO and rays exit at the top section of it. Nonuniform light distribution was obtained from this optic (see Figure 7 (b)). The reason of this non-uniform illuminance at the target plane is that section $\mathrm{S}_{1}$ received a light intensity 2.4 times higher than section $\mathrm{S}_{5}$ despite both sections having the same size. 
Table 1. Distribution of light flux from light source to each section of the first quarter of the target plane.

\begin{tabular}{|c|c|c|}
\hline Sections & Light receives & Percentages \\
\hline $\mathrm{S}_{1}$ & $18.4^{\circ}$ & $20.44 \%$ \\
\hline $\mathrm{S}_{2}$ & $15.3^{\circ}$ & $17 \%$ \\
\hline $\mathrm{S}_{3}$ & $11.3^{\circ}$ & $12.56 \%$ \\
\hline $\mathrm{S}_{4}$ & $8.1^{\circ}$ & $9 \%$ \\
\hline $\mathrm{S}_{5}$ & $7.54^{\circ}$ & $8.385 \%$ \\
\hline $\mathrm{S}_{6}$ & $8.8^{\circ}$ & $9.78 \%$ \\
\hline $\mathrm{S}_{7}$ & $9.94^{\circ}$ & $11.04 \%$ \\
\hline $\mathrm{S}_{8}$ & $10.62^{\circ}$ & $11.8 \%$ \\
\hline Sum & 90 degrees & $100 \%$ \\
\hline
\end{tabular}

(a)

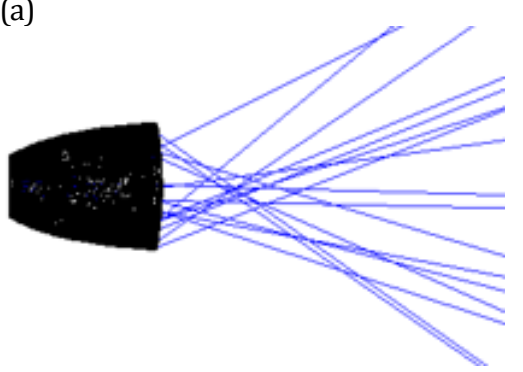

(b)

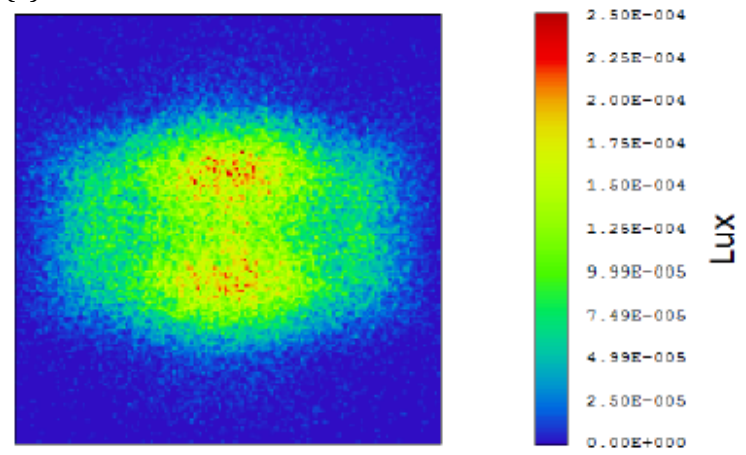

Fig. 7. RDTIRO-LED for rectangular footprint without optimisation (a) Ray tracing at the inside and outside of the optic. (b) Light distribution at the target plane by ZEMAX simulation.

\section{B. Optimisation process of the side profile of the RDTIRO}

The luminous flux has to be irradiated equally on the target plane in order to produce overall uniformity. Due to the fixed shape of the top section of the RDTIRO (as a part of a hemisphere), the light distribution of the first set of rays cannot be changed. Therefore, uniformity can only be obtained by optimising the side profile of the RDTIRO (which corresponds to the second set of rays).

First, the light intensity distribution corresponding to the two sets of rays has to be calculated. Then the size of the illuminated area of the second set of rays (the eight sections in the third quarter of Figure 6) has to be modified with respect to the corresponding luminous flux. For this it is necessary to calculate the amount of the luminous flux of two sets of rays for each section of the RDTIRO (or CDTIROs segments) by Equation 4 . This equation requires of two parts to find the amount of luminous flux ( $\Phi)$ : (i) the first part $\left(\Phi_{1}\right)$ corresponds to the first set of rays ( 0 to $\theta)$ and (ii) the second part $\left(\Phi_{2}\right)$ corresponds to the second set of rays $(\theta$ to $\phi)$ :
$\Phi=\int_{0}^{\phi} \int_{0}^{\delta} 2 \pi \sin \phi d \phi d \delta \sum_{0}^{\phi} \cos X=\Phi_{1}+\Phi_{2}=$

$\int_{0}^{\theta} \int_{0}^{\delta} 2 \pi \sin \theta d \theta d \delta \sum_{0}^{\theta} \cos X+\int_{\theta}^{\phi} \int_{0}^{\delta} 2 \pi \sin \phi d \phi d \delta \sum_{\theta}^{\phi} \cos X$

In equation $4, \delta$ can be calculated as follows:

$\delta_{1}=\operatorname{Arctan}\left(\frac{X_{1}}{Y_{14}}\right)$

The illumination of each section can be calculated by the following equation, $\mathrm{S}$ is the size of the illuminated surface area:

$E=\frac{\Phi}{S}$

(6)

For example, $E_{1}$ or $\frac{\Phi_{1}}{\mathrm{~s}_{1}}$ corresponds to the illumination of the first part of the luminous flux on the $S_{1}$.

According to Table 1 , sections $\mathrm{S}_{1}, \mathrm{~S}_{2}$ and $\mathrm{S}_{3}$ receive more than $50 \%$ of the total luminous flux. However, these three sections have $37.5 \%$ of the total area. In order to reduce the light intensity distribution on sections $\mathrm{S}_{1}{ }^{\prime}$ to $\mathrm{S}_{3}{ }^{\prime}$, these sections have to be expanded when the luminous flux remains unchanged. The expansion of sections $S_{1}$ ' to $S_{3}$ ' reduces the section area of $\mathrm{S}_{4}{ }^{\prime}$ to $\mathrm{S}_{6}{ }^{\prime}$ that increases the light intensity distribution on sections $\mathrm{S}_{4}{ }^{\prime}$ to $\mathrm{S}_{6}{ }^{\prime}$. Fig. 8 shows the new energy mapping of only the first quarter of the illumination system. Sections $S_{1}$ to $S_{8}$ are the target plane for the first set of rays, whereas sections $S_{1}{ }^{\prime}$ to $\mathrm{S}_{8}{ }^{\prime}$ are the target plane for the second set of rays. The first part of the illumination (only the refraction section on the hemisphere of the CDTIRO) is fixed, therefore the optimisation process cannot be carried out on the top of the CDTIRO. The optimisation process was only carried out for the second part of the illumination. Therefore, after the optimisation process, the sections of the first part of the illumination ( $S_{1}$ to $\left.S_{8}\right)$ remain unchanged, whilst the sections of the second part of illumination $\left(\mathrm{S}_{1}{ }^{\prime}\right.$ to $\left.\mathrm{S}_{8}{ }^{\prime}\right)$ were changed.

To increase uniformity, an iterative algorithm was used to change the position of the boundary of sections $S_{1}{ }^{\prime}$ to $S_{8}$ '. The calculation process was carried out by using Equations 4 and 5 . The border of the section $S_{1}$ ' to $S_{8}$ ' has to be changed until the ratio of the luminous flux and the corresponding section area is the same for all sections. The extreme ray of the first section (the ray with angle $\delta_{1}$ with respect to the OY-axis) has to be reflected and then refracted toward the new $\mathrm{R}_{2}$ ' line, which is the new boundary line between sections $\mathrm{S}_{1}{ }^{\prime}$ and $\mathrm{S}_{2}{ }^{\prime}$ (see Figure 8). Therefore, the light flux within the angle $\delta_{1}$ will be distributed on a new section $\mathrm{S}^{\prime}$.

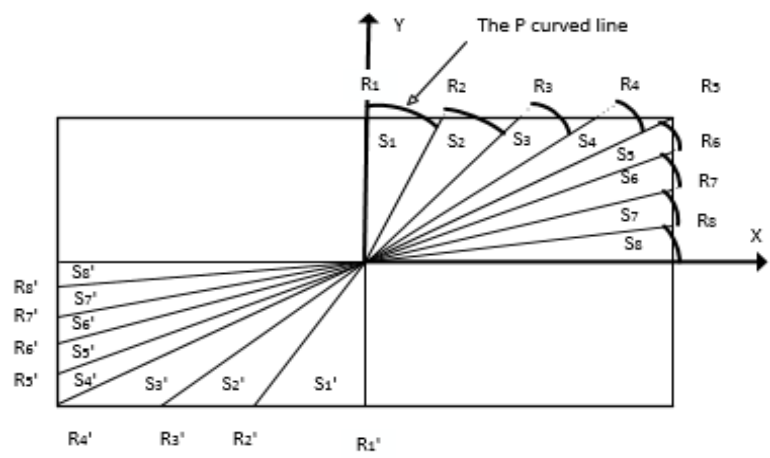


Fig. 8. Section $\mathrm{S}_{1}{ }^{\prime}, \mathrm{S}_{2}$ ' and $\mathrm{S}_{3}{ }^{\prime}$ are expanded in new energy mapping.

(a)

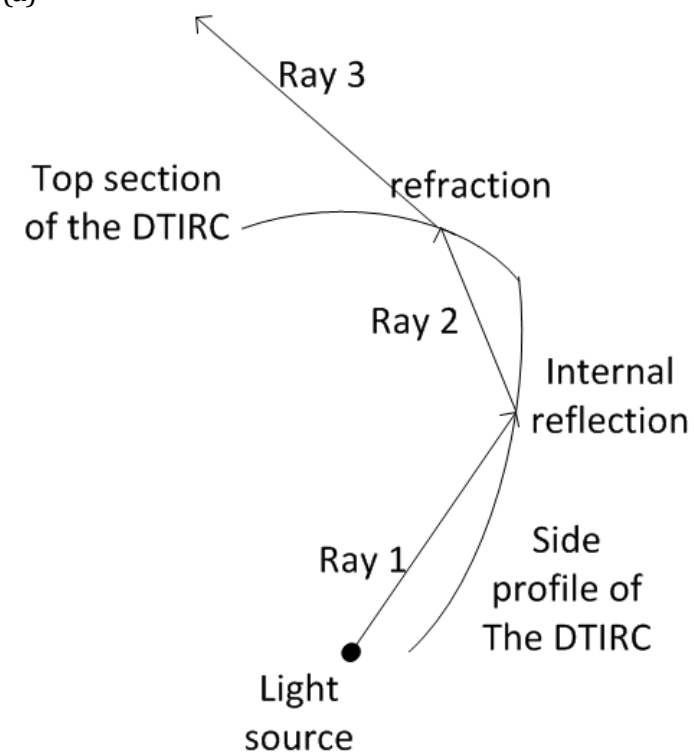

(b)

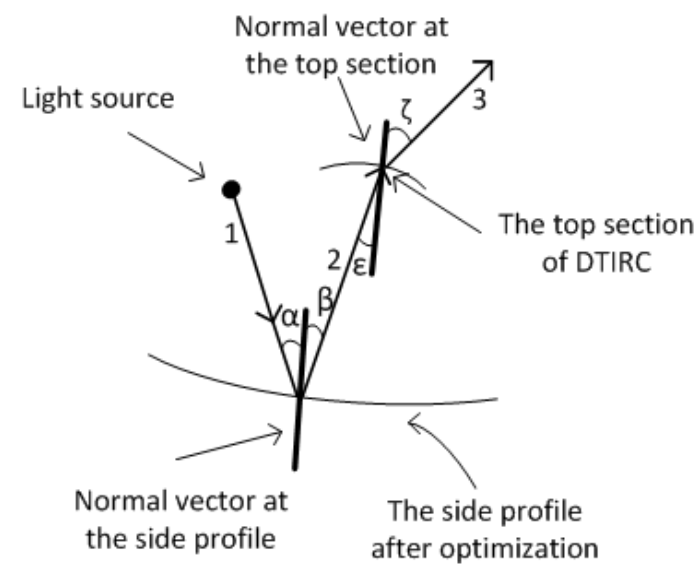

(c)

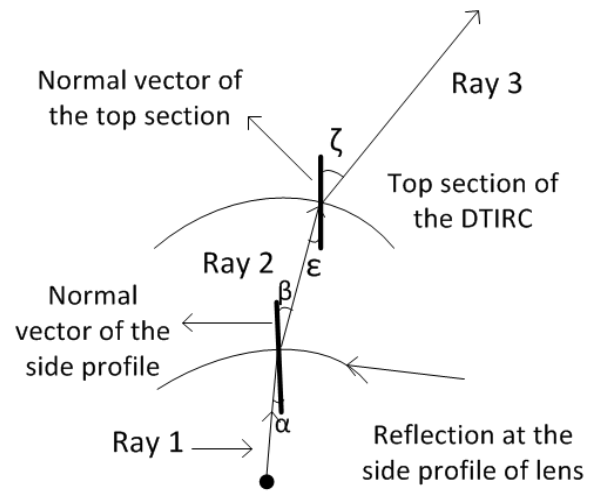

Fig. 9. Ray tracing for the optimisation of the side profile of the RDTIRO. a) Side view, b) Top view c) Back view.
The new $\mathrm{R}_{2}$ ' line was moved towards section $\mathrm{S}_{2}$ ' to expand section $S_{1}{ }^{\prime}$ as shown in Figure 8. In order to transfer the ray toward the new expanded area, the P curve (or the side profile of the lens) has to be tilted to change the direction of the normal vector on the side profile of the lens. This change modifies the angle between the emitted rays from the light source and the normal vector.

Figure 9 shows three different views of the ray tracing at the inside of the optic. Figure 9 (a) shows the ray tracing at the inside and outside of the optic from the cross section with the relevant normal vectors. On this diagram Ray 1 represents the ray emitted from the light source to the side profile of the optic and Ray 2 the ray reflected from the side profile of the lens toward the top section of the optic. Ray 3 represents the ray refracted to the target plane after refraction on the top section.

When Ray 2 reaches the top section of the optic, there is an angle between the Ray 2 and the normal vector of the top section of the lens after rotation of the side profile of the RDTIRO. Figure 9 (b) shows the $\varepsilon$ and $\zeta$ angles which are refraction angles. Angle $\varepsilon$ is the angle between Ray 2 and the normal vector at the inside of the optic; and angle $\zeta$ is the angle between ray 3 and the normal vector of the top section at the outside of the optic. Finally, the Ray 3 with an angle $\zeta$ forms the $\mathrm{R}_{2}{ }^{\prime}$ line at the target plane. If it does not reach the new target area (the new division of section $\mathrm{S}_{1}{ }^{\prime}$ ) it is possible to adjust the side profile of the RDTIRO. This modification changes the angle $\lambda$ and $v$ to refract the Ray 3 to the desired points with a new angle $\zeta$.

Figure 9 (c) also shows the $\varepsilon$ and $\zeta$ angles between Rays 2 and 3 with respect to the normal of the top section of the lens respectively. The process of rotation of the side profile of the lens was carried out until the extreme ray with angle $\delta$ of the first section formed the new line $\mathrm{R}_{2}$ ' on the new target plane. The same process has to be accomplished for other parts of the lens for transferring the light flux to the desired sections according to the new energy mapping. The relationship between the rays 2,3 and $\mathrm{N}(\mathrm{N}$ is the normal vector on the surface) is shown in Equation 7. These three vectors are related through Snell's laws:

$n_{o} \times$ Ray $3-n_{I} \times$ Ray $2=$
$\sqrt{\left[n_{o}^{2}+n_{I}^{2}-2 \times n_{I} \times n_{o} \times(\text { Ray } 2 \times \text { Ray } 3)\right]} \times N$

Where $n_{0}$ and $n_{I}$ indicate the refractive indices of the outside media and of the lens, respectively. $\mathrm{n}_{0}$ is assumed to be 1 for air.

Figure 10 shows the light distribution on the target plane that results from the use of the optimised RDTIRO with an LED. Figure 10 was obtained from the optical analysis software ZEMAX. Over 90\% uniformity was obtained within the rectangular area; this was achieved by optimising the side profile of the RDTIRO. The use of an extended light source leads to the creation of the transition illumination area between the uniformly illuminated area and the unilluminated area. The size of this section is directly proportional to the size of the light source with respect to the size of optic. Therefore, the transition area becomes larger by increasing the size of the light source or decreasing the size of the optic. There are two methods available to produce uniformity using an extended light source. Firstly, when the size of an optic is five times larger than the size of a light 
source, the light source acts as a point light source. However, if the size of the optic is not large enough to compensate for the extended light source, uniformity can be achieved by optimising the position of these desired points.

(a)

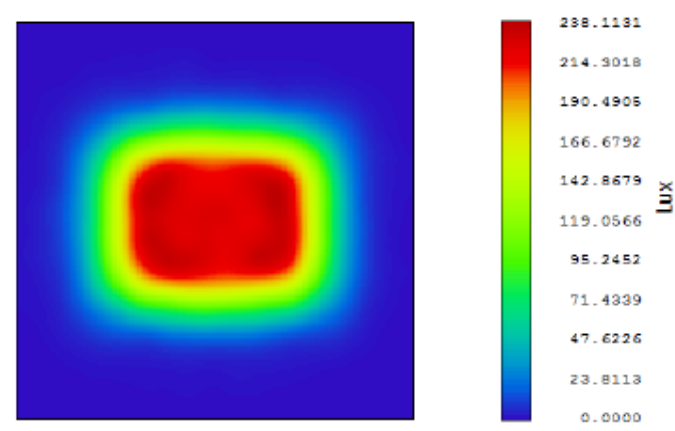

(b)

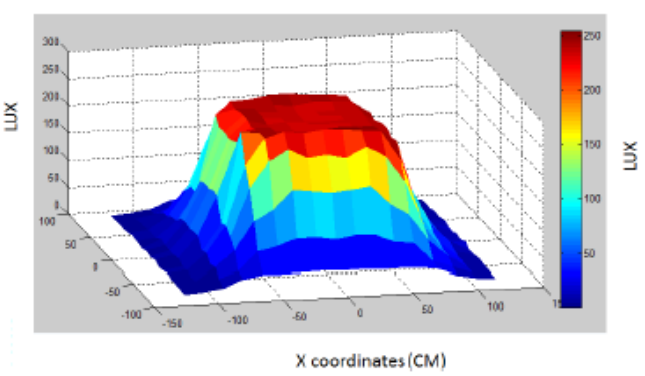

Fig. 10. Footprint of the RDTIR0 from software simulation: (a) Light distribution at the target plane. (b) 3-D Light distribution at the target plane.

\section{Illumination footprint from the RDTIRO prototype}

The illumination footprint of an RDTIRO prototype was obtained experimentally and compared to the one obtained from the software simulations. For the experimental work the same parameters (the same size of the light source, and optic) were as those used in the simulations were employed. Initially, the CDTIRO was designed based on a point source. However, an extended source was combined with the RDTIRO in the simulation and the experiment. Using an extended source does not affect the uniformly of illumination as long as the optic is sufficiently large with respect to the LED. The CREE Xlamp LED with $0.89 \mathrm{~cm}$ diameter and a Lambertian radiation pattern was used in this experiment. Figure 11 shows two photographs of the RDTIRO prototype. The computer numerical control (CNC) machining method was used to produce this RDTIRO due to its high flexibility of manufacturing. The polymethyl methacrylate (PMMA) material was chosen for manufacturing the RDTIRO. The PMMA material is a highly transparent thermoplastic polymer ( $92 \%$ transparent) with a 1.49 index of refraction at $589.3 \mathrm{~nm}$. The height, length and width of the optic are $32 \mathrm{~mm}, 40 \mathrm{~mm}$ and $30 \mathrm{~mm}$, respectively.

For the experiments the illumination system was located at 1 $m$ distance from the target plane. The size of the footprint and the light distribution obtained from the experimental work are very similar to the ones obtained from the simulations. Figure 12 shows the irradiance distribution of the LED-RDTIRO on the target plane. The light intensity was measured on the black marks on the target plane. The sensor of a lux meter was placed on the black marks facing towards the light source to take the lux measurements. Uniformity is defined as the ratio of the minimal illuminance over the area's weighted average illuminance ( $U=\frac{\text { Emin }}{\text { Eave }}$ ). Over of $90 \%$ uniformity was achieved by this optic. The phenomena that affects the optical efficiency of a lens can be organised into two groups. First group: absorption in the lens material, reflections at any of the lens faces, losses due a large number of grooves and their draft angles, high-angle Lambertian scattering at the interfaces, etc. Light affected by these parameters is simply lost. Second group: the characteristics that affect the spectral and spatial irradiance distribution at the lens focus: chromatic aberration, low-angle scattering due to surface quality, lack of flatness or other manufacturing errors, and temperature effects. The optical efficiency of the RDTIRO is over $90 \%$.

The RDTIRO was designed to be placed at the top in the centre of the illuminated area to produce a uniformly rectangular area. It has been designed for the Type I street lighting. It can also be used for other type patterns; however, uniformity will be reduced. A change in the top sections of the RDTIRO from a hemisphere to freeform surface is required in order to use the newly designed RDTIRO for the Type IV street lighting pattern. Maximum divergence of the output beam is larger than 60 degrees by using a freeform surface on the top of the RDTIRO.

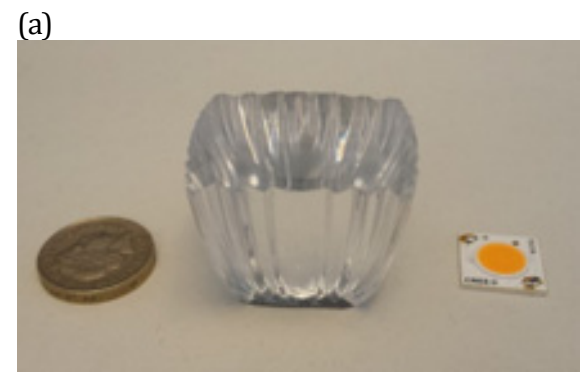

(b)

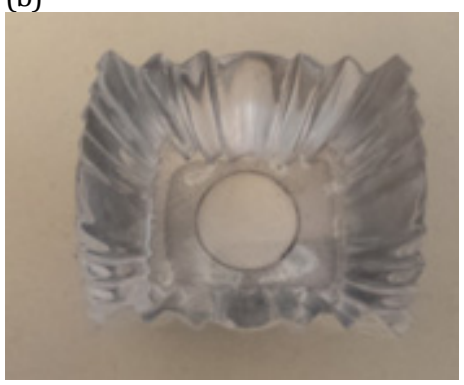

Fig. 11. RDTIRO Prototype: (a) Size comparison of the RDTIRO prototype with respect to the LED used and a $£ 1$ coin; (b) Top view of the RDTIRO prototype. 


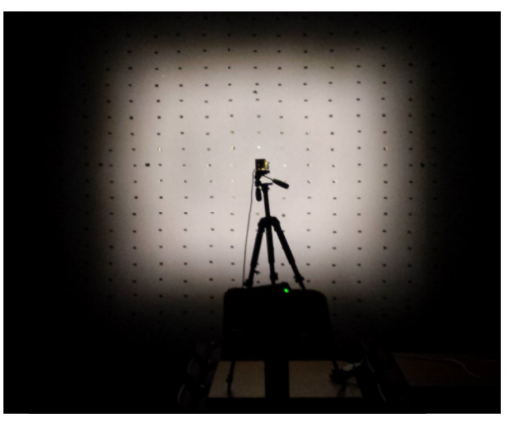

Fig. 12. Footprint of the RDTIRO-LED system at $1 \mathrm{~m}$ distance from the centre of the target plane.

\section{Conclusion}

In this research, a new energy mapping method has been proposed to produce an optic capable of providing uniform illuminance within a rectangular area. The illuminated area was irradiated via two sets of rays through the optic, the first set comprised of the rays refracted at the top section of the RDTIRO; and the second one of the rays reflected at the side profile and refracted at the top section of the lens. Uniformity was achieved by combining these two sets of rays. Ray tracing simulations and an experiment using the same parameters and conditions were carried out to investigate the illumination footprint produced by this optic. The results in both cases show uniformity of illumination of over $90 \%$.

Acknowledgment. This research was sponsored by the Energy Technology Partnership (ETP), Optical Antenna Solution (OAS) and Glasgow Caledonian University.

\section{References}

1. M. R. E. R. A. Pinto, M. R. Cosetin, M. F. da Silva, G. W. Denardin, A. Campos, and R. N. do Prado, "Led lamp with a compact emergency lighting system," in "Industry Applications Society Annual Meeting, 2009. IAS 2009. IEEE," pp. 1-6. (IEEE, 2009),

2. S. M. Ghoreyshi, A. Shahrabi, and T. Boutaleb, "An opportunistic void avoidance routing protocol for underwater sensor networks," in "Advanced Information Networking and Applications (AINA), 2016 IEEE 30th International Conference on," pp. 316-323. (IEEE, 2016).

3. S. Babadi, R. Ramirez-Iniguez, T. Boutaleb, and T. Mallick, "An optimisation of a freeform lens design for led street lighting," in "Students on Applied Engineering (ICSAE), International Conference for," pp. 89-92. (IEEE 2016).

4. J. Jiang, S. To, W. Lee, and B. Cheung, "Optical design of a freeform lens for led streetlight," Optik-International Journal for Light and Electron Optics 121, pp 1761-1765 (2010).

5. S. Babadi, R. Ramirez-Iniguez, T. Boutaleb, and T. Mallick, "Novel nonimaging optic design for uniform illumination," SPIE OPTO,(International Society for Optics and Photonics, 2016) p. 976416 (2016).

6. S. Babadi, R. Ramirez-Iniguez, T. Boutaleb, and T. Mallick, "Performance analysis of a DTIRC-led illumination structure," Optics and Measurement Conference 2014. Vol. 9442. International Society for Optics and Photonics, pp. 944205944205 (2014).

7. J. C. Minano, P. Benitez, J. C. Gonzalez, W. Falicoff, and H. Caulfield, "High efficiency non-imaging optics," (2003). US Patent 6,639,733.

8. S. J. C. Minano and J. P. P. Gonzalez, "Design of nonimaging lenses and lens-mirror combinations," in "Nonimaging Optics: Maximum
Efficiency Light Transfer," vol. 1528 (International Society for Optics and Photonics, 1991), vol. 1528, pp. 104-116.

9. C. Brecher, S. Lange, M. Merz, F. Niehaus, and M. Winterschladen, "Offaxis machining of nurbs freeform surfaces by fast tool servo systems," 4M 2006-Second International Conference on MultiMaterial Micro Manufacture. pp. 59-62. (2006).

10. Z. Zhenrong, H. Xiang, and L. Xu, "Freeform surface lens for led uniform illumination," Applied optics 48, 6627-6634 (2009).

11. C. Xu, C. Haobo and F. Yunpeng. "Optical design of rectangular illumination with freeform lenses for the application of LED road lighting." Frontiers of Optoelectronics 10.4 (2017): 353-362.

12. K. Wang et al. "Freeform LED lens for rectangularly prescribed illumination." Journal of Optics A: Pure and Applied Optics 11.10 (2009): 105501.

13. R. Winston, "Principles of solar concentrators of a novel design," Solar Energy 16, pp. 89-95 (1974).

14. R. Ramirez-Iniguez, A. Ahmadinia, and H. Fernandez-Canque, "DTIRC based optical collimators," Knowledge-Based and Intelligent Information and Engineering Systems pp. 462-471 (2011).

15. S. Babadi, R. Ramirez-Iniguez, T. Boutaleb, and T. Malick, "Novel dtircbased lens design for use with an extended light source for a rectangular footprint," in "Photonics Conference (IPC), 2016 IEEE," pp. 694-695. (IEEE, 2016).

16. F. Muhammad-Sukki, R. Ramirez-Iniguez, S. G. McMeekin, B. G. Stewart, and B. Clive, "Solar concentrators," International Journal of Applied Sciences 1, 1-15 (2010).

17. S. Babadi, R. Ramirez-Iniguez, T. Boutaleb, and T. Mallick, "Performance analysis of a DTIRC-led illumination structure," in "Optics and Measurement Conference 2014," vol. 9442 (International Society for Optics and Photonics, 2015), vol. 9442, p. 944205 (2014).

18. S. Babadi, R. Ramirez-Iniguez, T. Boutaleb, and T. Mallick, "Comparing freeform lenses and optimised DTIRC for uniform illumination," in "Photonics Conference (IPC), 2016 IEEE,", pp. 698-699. (IEEE, 2016) 